\title{
Large Spontaneous Coronary Artery Dissection Presenting as Acute STEMI - Is Intervention the Only Answer?
}

\author{
Santosh Kumar Sinha, FACC, FAESC, FSCAI* iD, Awadesh KumarSharma (D), Mahmodullah \\ Razi (D) and Umeshwar Pandey
}

Department of Cardiology, LPS Institute of Cardiology, GSVM Medical College, India

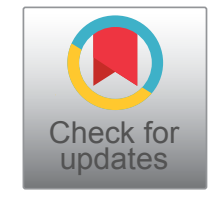

*Corresponding author: Santosh Kumar Sinha, FACC, FAESC, FSCAI, Associate Professor, Department of Cardiology, LPS Institute of Cardiology, GSVM Medical College, Kanpur, Uttar Pradesh, 208002, India, Tel: +91-9670220088, Fax: +910512-2556199; 2556521

\begin{abstract}
Spontaneous coronary artery dissection (SCAD) is a rare but an important cause of ST elevation myocardial infarction (STE$\mathrm{MI}$ ) in young patients. Here, we report case of a 32-year-old male presenting as acute anterior wall STEMI for which was thrombolysed. Coronary angiogram revealed spiral luminal filling defects with extensive contrast staining of proximal left anterior descending artery involving diagonal branch representing Type $D$ dissection. As patient refused any intervention, he was managed conservatively. He presented with unstable angina 6-weeks later. Repeat angiogram revealed complete healing of dissection along with borderline lesion. As his treadmill test was negative for exercise induced myocardial ischaemia, he was managed conservatively. This case highlights that beside intervention, patients with high grade dissection may respond to conservative management.
\end{abstract}

\section{Keywords}

Spontaneous coronary artery dissection, Myocardial infarction, Myocardial ischaemia

\section{Introduction}

Spontaneous coronary artery dissection (SCAD) is a rare but an important cause of acute coronary syndromes which has a striking predilection for women. Clinical presentation ranges from asymptomatic state to acute coronary syndrome, ventricular arrhythmias, heart failure and sudden cardiac death [1]. Predisposing factors include atherosclerosis, peripartum period, structural and inflammatory conditions affecting arterial wall or extension of aortic dissection [2]. In interventional era, early angiography and primary percutaneous coronary angioplasty ( $\mathrm{pPCl}$ ) has led to earlier identification of patients with SCAD and may encourage an increased use of various revascularization strategies [2]. Prompt diagnosis and treatment of patients with dissection improves survival. Management of SCAD sometime can be challenging. Various treatment options have been utilized, including conservative management, $\mathrm{PCl}$, and coronary artery bypass graft surgery (CABG) though primary $\mathrm{PCl}$ remains the reperfusion strategy of choice.

\section{Case Report}

A 32-year-old apparently healthy male presented with excruciating chest pain with diaphoresis of 2-hour duration. His pulse rate was 85 beats/min and blood pressure was $110 / 84 \mathrm{mmHg}$. Other physical findings were all normal. Electrocardiogram showed normal sinus rhythm and ST-elevation in $\mathrm{V}_{1}-\mathrm{V}_{6}$ with reciprocal changes in inferior leads. An echocardiogram revealed regional wall motion abnormality in left anterior descending artery territory (LAD) with mild left ventricular (LV) systolic dysfunction (ejection fraction $=45 \%$ ). His routine blood examinations were normal. He was loaded with aspirin $300 \mathrm{mg}$, clopidogrel $300 \mathrm{mg}$ and thrombolysed with $40 \mathrm{mg}$ Tenecteplase. At 90 minute, chest pain was persistent with little resolution of ST segment ( $\leq 50 \%$ from initial elevation) which indicated failed lyses. Coronary angiography was performed through transfemoral after obtaining written consent. LAD showed large, spontaneous spiral dissection with flap beginning from osteo-proximal segment exten-

Citation: Sinha SK, Sharma AK, Razi M, Pandey U (2021) Large Spontaneous Coronary Artery Dissection Presenting as Acute STEMI - Is Intervention the Only Answer?. Int J Clin Cardiol 8:219. doi. org/10.23937/2378-2951/1410219

Accepted: February 25, 2021: Published: February 27, 2021

Copyright: (C) 2021 Sinha SK, et al. This is an open-access article distributed under the terms of the Creative Commons Attribution License, which permits unrestricted use, distribution, and reproduction in any medium, provided the original author and source are credited. 


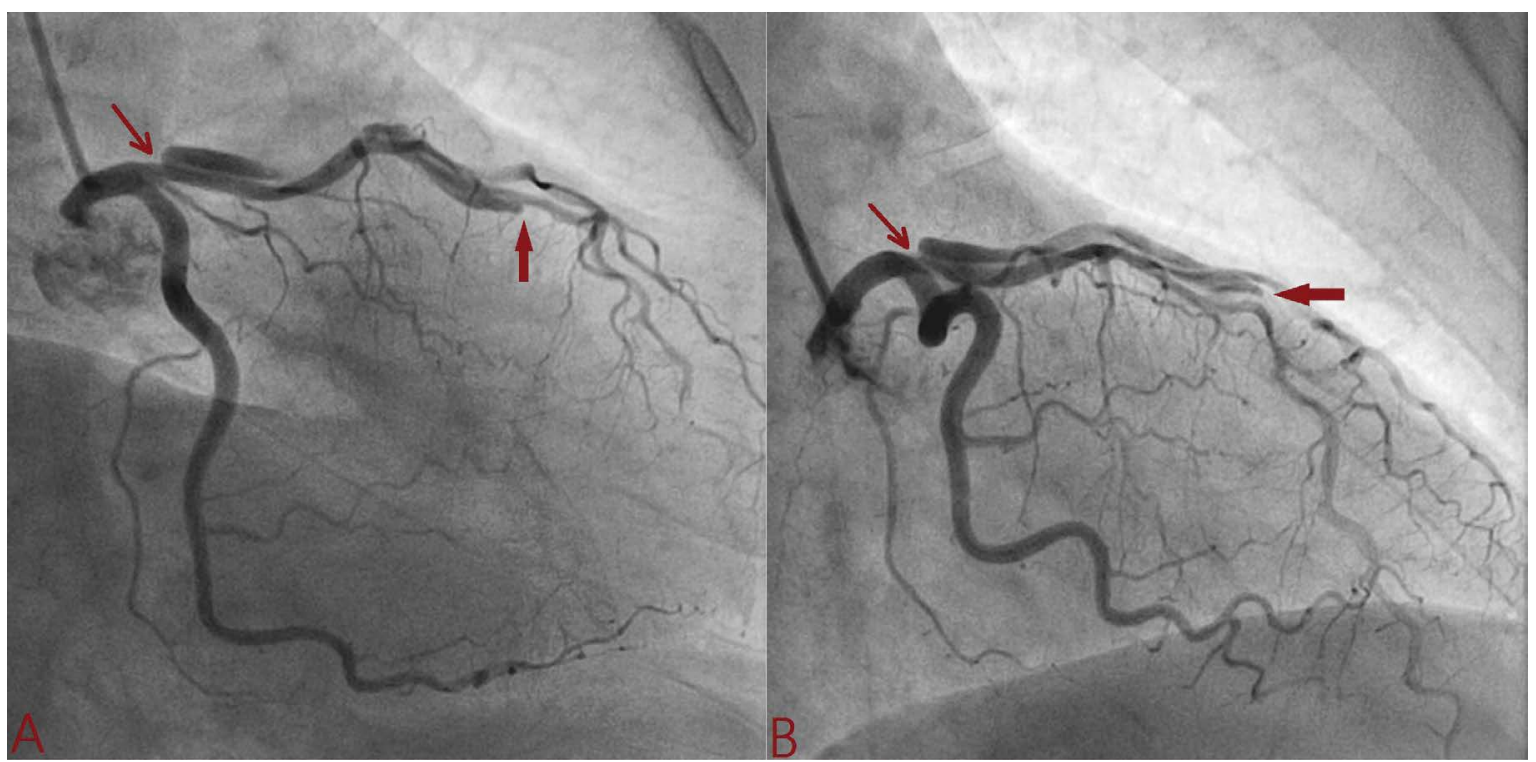

Figure 1: Spiral dissection extending from osteo-proximal left anterior descending artery involving its large diagonal branch (A: Antero-posterior caudal view; B: Right anterior oblique caudal view. Red arrow indicate intimal flap).

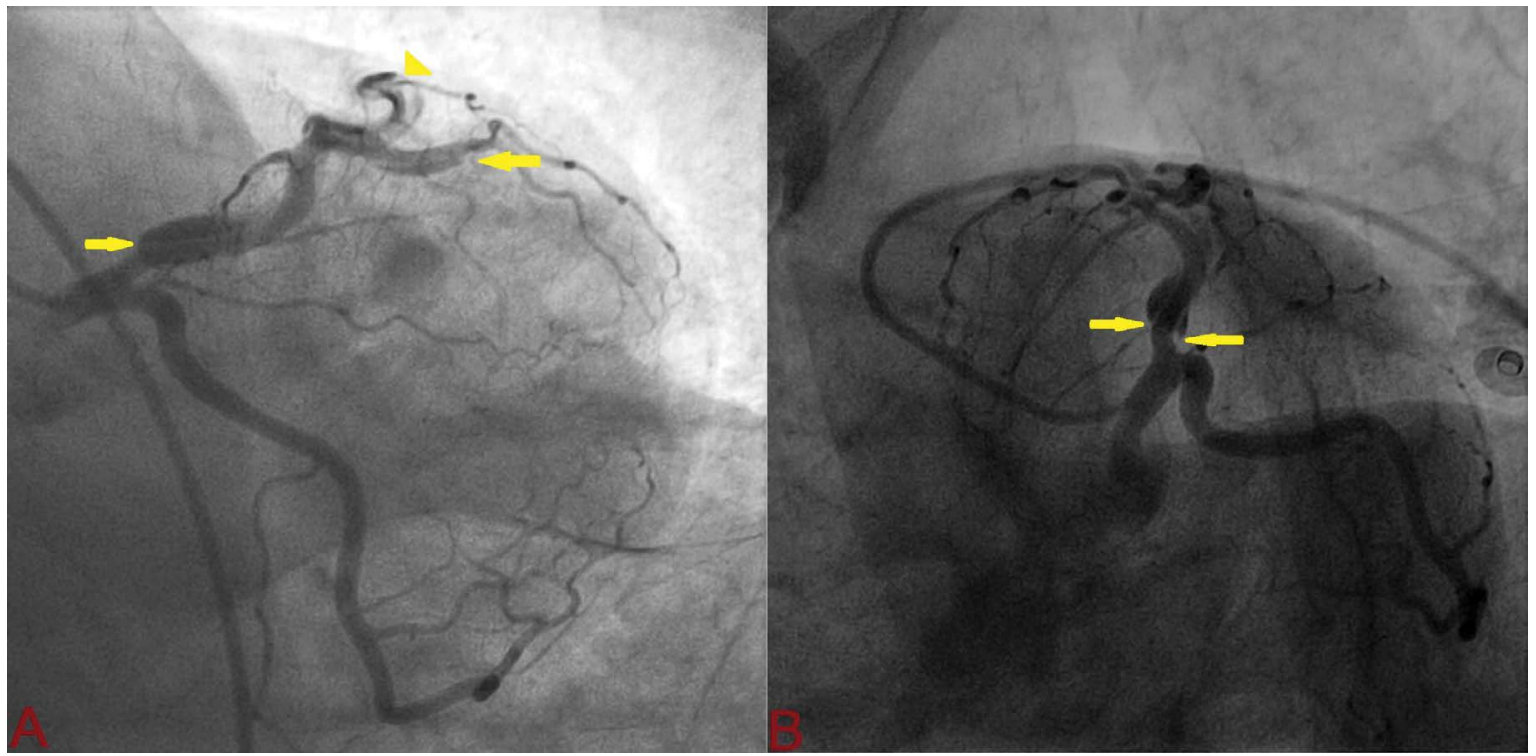

Figure 2: Spiral dissection beginning from osteo-proximal left anterior descending artery involving large diagonal branch (A: Left anterior oblique shallow view; B: Spider view. Yellow arrow indicate intimal flap).

ding up to mid segment involving first diagonal branch which was labelled as Type-D SCAD (Figure 1 and Figure 2). As the origin of dissection flap was appreciated on first injection and originating away from the ostial segment of LAD, it ruled out iatrogenic dissection. The rest of his coronary trees were normal. $\mathrm{PCl}$ was advised $\mathrm{PCl}$ but patient refused. He was conservatively managed with Ecospirin $75 \mathrm{mg}$, Clopidogrel $75 \mathrm{mg}$, Rosuvastatin $10 \mathrm{mg}$, Ramipril $5 \mathrm{mg}$ and Metoprolol $100 \mathrm{mg}$. Repeat angiogram 4-weeks later revealed complete healing of dissection along with borderline lesion (Figure 3). Echocardiography indicated normalization of systolic function (ejection fraction-70\%). As his treadmill test was negative for exercise induced myocardial ischaemia, he was managed conservatively.

\section{Discussion}

Spontaneous coronary artery dissection is defined as non-traumatic, non-iatrogenic separation of intima and media as a result of either intimal tearing and medial haemorrhage or spontaneous rupture of vaso-vasorum and bleeding into arterial wall causing formation of a false lumen which may lead to compression of true lumen [3]. It may lead to lead to acute coronary syndrome (ACS) primarily due to formation of an intramural hematoma and subsequent luminal narrowing, cardiac arrest, heart failure, and death especially in young women who do not have traditional cardiovascular risk factors. Most of ACS is STEMI in $33-87 \%$ and NSTEMI in $13-67 \%$ of cases [4]. Coronary angiography is the gold standard modality of diagnosis. It has a predilection for mid-to- 


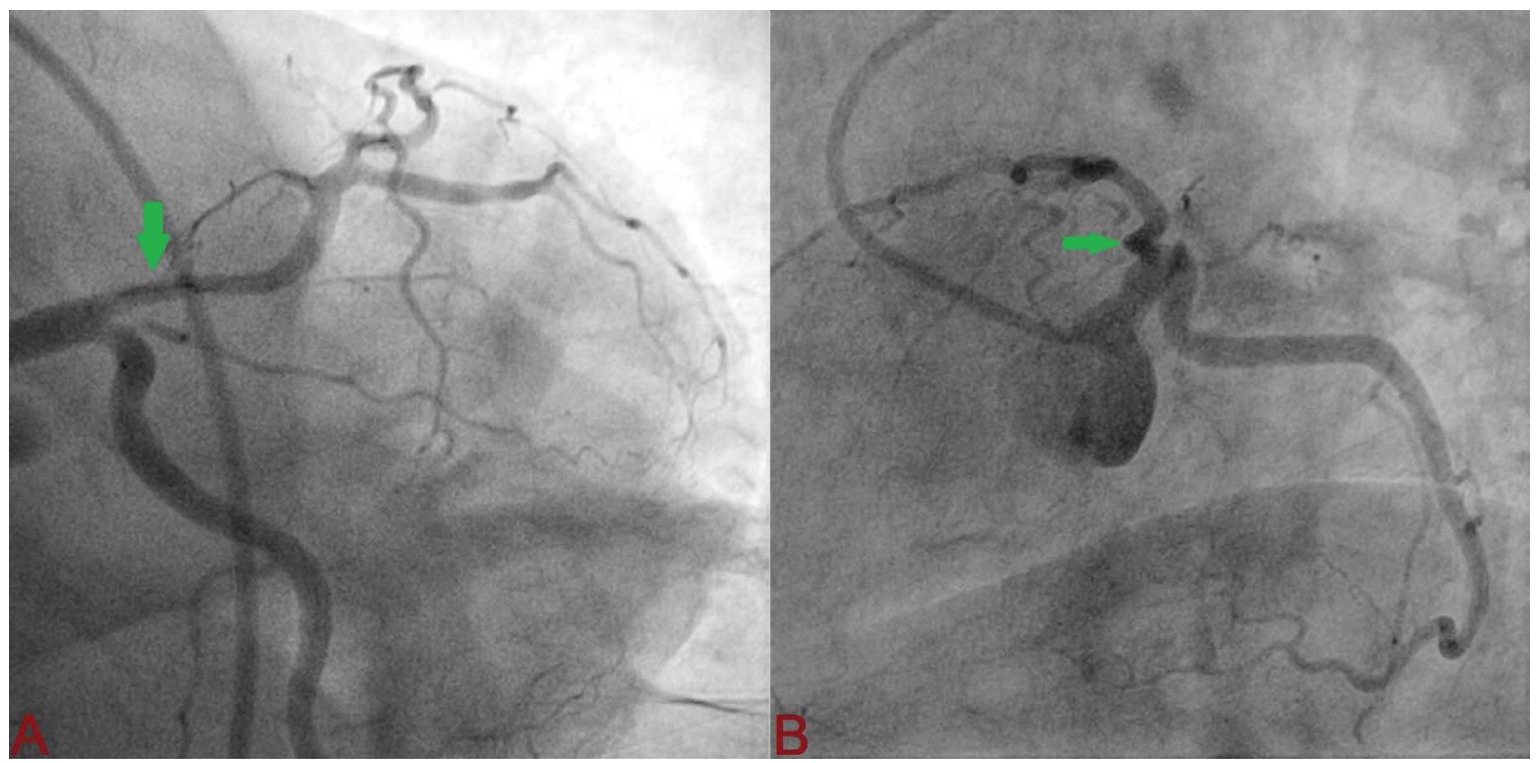

Figure 3: Complete sealing of intimal flap (A: Antero-posterior caudal view; B: Spider oblique caudal view. Green arrow indicate sealed flap).

distal coronary arteries with LAD being most commonly involved [5].

Dissection in SCAD is usually characterized by a long smooth narrowing which tapers distally with either distal reconstitution of a normal vessel or extending into terminal branches. Presence of tortuosity and lack of intraluminal thrombus helps to differentiate it from dissection resulting from rupture of atherosclerotic plaque [6]. Chest pain after SCAD is not infrequent and may be either recurrent or may persist for weeks often leading to frequent hospitalization [7]. Mental stress, exertion, and perimenopausal state are few of the triggers. It may be present despite absence of fixed coronary obstruction or inducible ischemia on functional testing (tread mill, dobutamine stress, stress thallium scintigraphy). The primary aim is restoration of TIMI (Thrombolysis in Myocardial Infarction) grade 3 flows, function and prevention of recurrence. Conservative management is mainstay of treatment for SCAD in absence of high-risk features. There is no consensus regarding optimal strategy to manage SCAD as randomized control trials are lacking. Optimal medical treatment includes long term aspirin and beta-blockers. Beta-blockers have been shown to reduce its recurrence [8]. Management is decided by risk stratification. Those having left main dissection, persistent or recurrent ischemic, ventricular tachycardia, cardiogenic shock and mechanical complication like mitral regurgitation are labelled as high risk [9] in this subgroup of patients, revascularization by either coronary artery bypass (CABG) or $\mathrm{PCl}$ is deemed appropriate [10].

$\mathrm{PCI}$ for SCAD is associated with less success rate (46\%) and increased complication. Increased risk of iatrogenic dissection and progression of intramural hematoma may further worsen luminal narrowing and ischemia. In the largest study (CanSCAD study) involving 750 patien- ts, $87 \%$ patients were managed initially with a conservative strategy, $13 \%$ underwent $\mathrm{PCl}$ while $1 \%$ underwent CABG or fibrinolysis [11]. In the conservative arm, only $2 \%$ required subsequent $\mathrm{PCl}$ and $0.3 \%$ underwent $\mathrm{CABG}$ while those who had undergone $\mathrm{PCl}$, only $30 \%$ were successful [11]. $\mathrm{PCl}$ is associated with an increased risk of, iatrogenic dissection, abrupt vessel closure, and hematoma propagation requiring implantation of multiple stents. Once the haematoma regresses, it may complicate into late stent malapposition. When $\mathrm{PCl}$ is deemed necessary, use of small-diameter balloon, or cutting balloon/scoring balloon angioplasty to fenestrate and depressurize the false lumen, proximal and distal edge stenting, and use of bioresorbable vascular scaffolds are advocated. As majority of haematoma regress within 30 days, it is recommended as optimal cooling period for SCAD [12]. CABG is also associated with some technical issues as dissected coronary artery tissues are very fragile and prone to anastomotic complications as placement of suture is often difficult. If the flap is extending much distally, it is a poor candidate for CABG like in our case. In a series of 20 patients who underwent CABG for SCAD by Tweet, et al. $5 \%$ mortality was observed and graft patency was 33\% at median follow-up of 3.5 years [13]. It may be attributed to increased competitive flow between native coronary arteries following healing and the conduits. Thrombolysis is associated with extension of dissection or hematoma, and therefore not recommended. Another study involving 24 patients following SCAD who were followed up using coronary CT angiogram for 4 months, twenty (88\%) had complete resolution while three had persistent dissection, aneurysm formation and one case had PCl in the false lumen [14]. Those who undergoes $\mathrm{PCl}$, dual antiplatelet should be continued as per standard guideline ( $\geq 1$ year) while those who are medically managed, addition of second P2Y12 inhibitor is debatable. The duration may be $\geq 1$ 
year as per usual ACS guidelines or may be limited to few months [10].

\section{Sources of Support (If Applicable)}

Disclosure of funding received for this work from any of the following organizations: National Institutes of Health (NIH); Welcome Trust; and other(s) - None.

\section{Statement of Equal Authors' Contribution}

All authors made equal contribution.

\section{References}

1. Hayes SN, Kim ESH, Saw J, Adlam D, Arslanian-Engoren C, et al. (2018) Spontaneous coronary artery dissection: Current state of the science: A scientific statement from the American Heart Association. Circulation 137: e523-e557.

2. Adlam D, Alfonso F, Maas A, Vrints C (2018) European Society of Cardiology, acute cardiovascular care association, SCAD Study Group: A position paper on spontaneous coronary artery dissection. Eur Heart J 39: 3353-3368.

3. Saw J, Mancini GBJ, Humphries K, Fung A, Boone R, et al (2016) Angiographic appearance of spontaneous coronary artery dissection with intramural hematoma proven on intracoronary imaging. Catheter Cardiovasc Interv 87: E54-E61.

4. Rogowski S, Maeder MT, Weilenmann D, Haager PK, Ammann P, et al. (2017) Spontaneous coronary artery dissection: Angiographic follow-up and long-term clinical outcome in a predominantly medically treated population. Catheter Cardiovasc Interv 89: 59-68.

5. Hayes SN, Tweet MS, Adlam D, Kim ESH, Gulati R, et al. (2020) Spontaneous coronary artery dissection: JACC State-of-the-Art Review. J Am Coll Cardiol 76: 961-984.

6. Duran JM, Naderi S, Vidula M, Michalak N, Chi G, et al. (2020) Spontaneous coronary artery dissection and its as- sociation with takotsubo syndrome: Novel insights from a tertiary center registry. Catheter Cardiovasc Interv 95: 485491.

7. Gad MM, Mahmoud AN, Saad AM, Bazarbashi N, Ahuja $\mathrm{KR}$, et al. (2020) Incidence, clinical presentation, and causes of 30-day readmission following hospitalization with spontaneous coronary artery dissection. JACC Cardiovasc Interv 13: 921-932.

8. Saw J, Humphries K, Aymong E, Sedlak T, Prakash R, et al. (2017) Spontaneous coronary artery dissection: Clinical outcomes and risk of recurrence. J Am Coll Cardiol 70: 1148-1158.

9. Saw J, Mancini GBJ, Humphries KH (2016) Contemporary review on spontaneous coronary artery dissection. J Am Coll Cardiol 68: 297-312.

10. Yang C, Alfadhel M, Saw J (2020) Spontaneous coronary artery dissection: Latest developments and new frontiers. Curr Atheroscler Rep 22: 49-53.

11. Saw J, Starovoytov A, Humphries K, Sheth T, So Derek, et al. (2019) Canadian spontaneous coronary artery dissection cohort study: In-hospital and 30-day outcomes. Eur Heart J 40: 1188-1197.

12. Hassan S, Prakash R, Starovoytov A, Saw J (2019) Natural history of spontaneous coronary artery dissection with spontaneous angiographic healing. JACC Cardiovasc Interv 12: 518-527.

13. Tweet MS, Eleid MF, Best PJM, Lennon RJ, Lerman A, et al. (2014) Spontaneous coronary artery dissection: Revascularization versus conservative therapy. Circ Cardiovasc Interv 7: 777-786.

14. Roura G, Ariza-Solé A, Rodriguez-Caballero IF, Gomez-Lara J, Ferreiro JL, et al. (2016) Noninvasive follow-up of patients with spontaneous coronary artery dissection with CT angiography. JACC Cardiovasc Imaging 9: 896-897. 